\title{
ON SYMMETRY IN CONVEX TOPOLOGICAL VECTOR SPACES
}

\author{
SETH WARNER AND ALEXANDER BLAIR
}

A convex topological vector space $E$ is called symmetric if and only if the topology of the strong bidual of $E$ induces on $E$ its given topology. A barrel in $E$ is a closed, convex, equilibrated, absorbing set (see [1] for the terminology); $E$ is called barrelled (French: espace tonnelé) if and only if every barrel is a neighborhood of 0 . The properties of being symmetric and of being barrelled are but the two extreme examples of a family of properties which we first wish to discuss. Second, we give a new counter-example in the theory of convex topological vector spaces. All spaces considered are assumed Hausdorff.

1. If $E$ and $F$ are convex topological vector spaces, $E^{\prime}$ the topological dual of $E, \Sigma$ a class of bound subsets of $E$ such that $U[S \mid S \in \Sigma]$ $=E$, then $E_{\Sigma^{\prime}}$ [respectively $\mathcal{L}_{\Sigma}(E, F)$ ] denotes the vector space $E^{\prime}$ [respectively $\mathcal{L}(E, F)$, the vector space of all continuous linear transformations from $E$ into $F$ ] with the (convex) topology of uniform convergence on all members of $\Sigma$. For the important special case where $\Sigma$ is all bound subsets, we write " $b$ " for " $\Sigma$ "; for that where $\Sigma$ is all one-point subsets of $E$, we write " $s$ " for " $\Sigma$." If $E^{\prime}$ is a total subspace of the algebraic dual of $E$, among all convex topologies on $E$ yielding $E^{\prime}$ as dual there is a strongest, denoted by $\tau\left(E, E^{\prime}\right)$ [5, Theorem 5]; if a given convex topological vector space $E$ with dual $E^{\prime}$ has the topology $\tau\left(E, E^{\prime}\right)$, it is called relatively strong.

If $E$ is a convex topological vector space, $E^{\prime}$ its dual, $\Sigma$ a class of bound subsets of $E$ such that $U[S \mid S \in \Sigma]=E$, then $E$ may be canonically identified (algebraically) with a subspace of the vector space (without topology) $\left(E_{\Sigma}^{\prime}\right)^{\prime}$. Since $L \subseteq E^{\prime}$ is equicontinuous if and only if the polar of $L$ in $E$ is a neighborhood of 0 , it is easy to see that if $\Lambda$ is a class of bound subsets of $E_{\Sigma}^{\prime}$ such that $U[L \mid L \in \Lambda]$ $=E^{\prime}$, then the topology induced on $E$ by that of $\left(E_{\Sigma}^{\prime}\right)_{\Lambda}^{\prime}$ is the given topology of $E$ if and only if $\left(E_{\Sigma}^{\prime}\right)_{\Lambda}^{\prime}=\left(E_{\Sigma}\right)_{\Omega}^{\prime}$, where $\Omega$ is the class of all equicontinuous subsets of $E^{\prime}$. Hence, since every equicontinuous subset of $E^{\prime}$ is bound in $E_{\Sigma}^{\prime}$, the topology of $\left(E_{\Sigma}^{\prime}\right)_{b}^{\prime}$ always induces on $E$ a stronger (i.e., at least as strong) topology than the given topology.

Definition. $E$ is $\Sigma$-symmetric if and only if the topology induced

Received by the editors June 7, 1954. 
on $E$ by that of $\left(E_{\Sigma}^{\prime}\right)_{b}^{\prime}$ is the given topology of $E$.

TheOREM 1. If $\Lambda$ is a class of bound subsets of $E$ such that $\Lambda \supseteq \Sigma$ and if $E$ is $\Sigma$-symmetric, then $E$ is $\Lambda$-symmetric.

Proof. As the topology of $E_{\Delta}^{\prime}$ is stronger than that of $E_{\Sigma}^{\prime}$, there are fewer bound sets in $E_{\Lambda}^{\prime}$ than in $E_{\Sigma}^{\prime}$; hence the topology of $\left(E_{\Lambda}^{\prime}\right)_{b}^{\prime}$ is weaker than the topology of $\left(E_{\Sigma}^{\prime}\right)_{b}^{\prime}$, and hence must also induce on $E$ its given topology.

Theorem 1 shows that among all the properties of being $\Sigma$-symmetric, symmetry (i.e., $b$-symmetry) is the weakest. The strongest such property is $s$-symmetry (i.e., $\Sigma$-symmetry where $\Sigma$ is the class of all one-point subsets of $E$ ). Theorem 2 shows that this property is precisely the property of being barrelled.

Theorem 2. Let $E$ and $F$ be convex topological vector spaces, $F$ of nonzero dimension, $\Sigma$ a class of bound subsets of $E$ such that $U[S \mid S \in \Sigma]$ $=E$. Then the following are equivalent: (1) $E$ is $\Sigma$-symmetric. (2) The polars in $E$ of all bound subsets of $E_{\Sigma}^{\prime}$ form a fundamental system of neighborhood of 0 for the topology of $E$. (3) Every bound subset of $E_{\Sigma}^{\prime}$ is equicontinuous. (4) Every bound subset of $\mathcal{L}_{\Sigma}(E, F)$ is equicontinuous. (5) Every barrel in $E$ absorbing all members of $\Sigma$ is a neighborhood of 0. (6) $E$ is relatively strong, and every convex bound subset of $E_{\Sigma}^{\prime}$ has compact closure in $E_{s}^{\prime}$.

Proof. The equivalence of (1), (2), and (3) follows immediately from our discussion above. The equivalence of (3) and (5) follows from the fact that a set is bound in $E_{\Sigma}^{\prime}$ if and only if its polar in $E$ is a barrel absorbing all members of $\Sigma$. Proposition 2 of [3] asserts the equivalence of (5) and (6) for the special case of barrelled spaces, and Theorem 1 of [3] asserts that (5) implies (4) for barrelled spaces. In both cases an obvious modification of the proof yields the desired result. It remains to show that (4) implies (3). Let $K y_{0}$ be a onedimensional subspace of $F, K$ the scalar field. $\phi: \lambda y_{0} \rightarrow \lambda$ is a topological isomorphism from $K y_{0}$ onto $K$. Let $L=\left[u \in \mathcal{L}(E, F) \mid u(E) \subseteq K y_{0}\right]$ with the topology induced from $\mathcal{L}_{\Sigma}(E, F)$. It is immediate that $\psi$ : $u \rightarrow \phi \bigcirc u$ is a topological isomorphism from $L$ onto $E_{\Sigma}^{\prime}$. Let $B$ be bound in $E_{\Sigma}^{\prime}, V$ a neighborhood of 0 in $K$. Then $V y_{0}=W \cap K y_{0}$ where $W$ is a neighborhood of 0 in $F \cdot \psi^{-1}(B)$ is bound in $L$, hence in $\mathcal{L}_{\Sigma}(E, F)$, and hence is an equicontinuous subset of $\mathcal{L}(E, F)$. Therefore there exists a neighborhood $W^{\prime}$ of 0 in $E$ such that if $u \in \psi^{-1}(B)$ then $u\left(W^{\prime}\right) \subseteq W$ and hence, as $u \in L, u\left(W^{\prime}\right) \subseteq W \cap K y_{0}=V y_{0}$. But then if $v \in B, \phi^{-1} \bigcirc v \in \psi^{-1}(B)$ so $\phi^{-1}\left(v\left(W^{\prime}\right)\right) \subseteq V y_{0}$, i.e., $v\left(W^{\prime}\right) \subseteq \phi\left(V y_{0}\right)$ $=V$. Hence $B$ is equicontinuous. 
CoROLlary 1. A necessary and sufficient condition that $E$ be barrelled is that $E$ be $\Sigma$-symmetric and that every bound subset of $E_{\text {s }}^{\prime}$ be bound in $E_{\Sigma}^{\prime}$.

"Sequentially complete" (i.e., all Cauchy sequences converge) can replace "complete" in Theorem 2 of [3]; hence

Corollary 2. If every $S \in \Sigma$ is sequentially complete, then $E$ is barrelled if and only if $E$ is $\Sigma$-symmetric.

It is obvious from Theorem 2 that for every theorem about barrelled spaces there is an analogue for $\Sigma$-symmetric spaces. We mention in particular the abstract version of the Banach-Steinhaus theorem, the proof of which for barrelled spaces is found in [2] or in Corollaries 1 and 2 of Theorem 1 of [3].

TheOREM 3. Let $E$ be $\Sigma$-symmetric, $\Phi$ a filter on $\mathcal{F}(E, F)$, the vector space of all functions from $E$ into $F, u_{0} \in \mathcal{F}(E, F)$. Then $u_{0} \in \mathcal{L}(E, F)$ and $\Phi$ converges to $u_{0}$ in the topology of uniform convergence on all precompact subsets of $E$ under any of the following additional assumptions: (1) $\Phi$ contains a bound subset of $\mathcal{L}_{\Sigma}(E, F)$ and $\Phi$ converges pointwise to $u_{0}$; (2) $F$ is quasi-complete (i.e., every closed, bound subset of $F$ is complete), $\Phi$ contains a bound subset of $\mathcal{L}_{\Sigma}(E, F)$, and $\Phi$ converges pointwise to $u_{0}$ on a total subset of $E$.

2. $E$ is called semi-reflexive if $\left(E_{b}^{\prime}\right)^{\prime}=E$ (algebraically). $E$ is called boundedly closed if every bound linear functional on $E$ is continuous. By Theorem 2, a symmetric space is relatively strong. We show the converse is false by giving an example of a semi-reflexive, relatively strong space $F$ whose strong dual $F_{b}^{\prime}$ is a Banach space, but which is neither symmetric nor boundedly closed.

Let $E$ be a nonreflexive Banach space (e.g., $L^{1}$ of the unit interval). $E$ may be regarded as a total subspace of the algebraic dual of $E^{\prime}$; we let $F$ be the vector space $E^{\prime}$ together with the convex topology $\tau\left(E^{\prime}, E\right) . F$ is thus by definition relatively strong. We show $F_{b}^{\prime}=E$ (algebraically and topologically). Since $E$ is barrelled, the classes of all bound subsets of $E_{s}^{\prime}$, of all bound subsets of $E_{b}^{\prime}$, and of all equicontinuous subsets of $E^{\prime}$ are identical (Theorem 2); this class is also identical with the class of all bound subsets of $F$, since $E$, the topological dual of $F$, is also the topological dual of $E_{s}^{\prime}[4$, Theorem 2], and hence $F$ and $E_{s}^{\prime}$ have the same bound subsets [5, Theorem 7]. $V$ is a neighborhood of 0 in $F_{b}^{\prime}$ if and only if $V$ contains the polar of a bound subset of $F ; V$ is a neighborhood of 0 in $E$ if and only if $V$ contains the polar of an equicontinuous subset of $E^{\prime}$; hence $E$ is 
topologically and algebraically identical with $F_{b}^{\prime}$. Thus $\left(F_{b}^{\prime}\right)^{\prime}=E^{\prime}=F$, so $F$ is semi-reflexive. As $E$ is not reflexive, $\left(E_{b}^{\prime}\right)^{\prime}$ strictly contains $E$. The topology of $\left(F_{b}^{\prime}\right)_{b}^{\prime}=E_{b}^{\prime}$ is thus strictly stronger than that of $F$ since $F$ and $\left(F_{b}^{\prime}\right)_{b}^{\prime}$ have different topological duals; hence $F$ is not symmetric. Also, as the bound subsets of $F$ and of $E_{b}^{\prime}$ coincide, every linear functional in $\left(E_{b}^{\prime}\right)^{\prime}$ is bound on $F$, but as $F^{\prime}=E \neq\left(E_{b}^{\prime}\right)^{\prime}$, there exist bound linear functionals on $F$ which are not continuous. Hence $F$ is not boundedly closed.

\section{BIBLIOGRAPHY}

1. N. Bourbaki, Espaces vectoriels topologiques, Chaps. I-II, Actualités Scientifiques et Industrielles, no. 1189, Paris.

2. - E E E - Espaces vectoriels topologiques, Chaps. III-V, to be published soon.

3. - Sur certains espaces vectoriels topologiques, Ann. Inst. Fourier, Grenoble vol. 2 (1950) pp. 5-16 (1951).

4. J. Dieudonné, La dualité dans les espaces vectoriels topologiques, Ann. École Norm. (3) vol. 59 (1942) pp. 107-139.

5. G. W. Mackey, On convex topological linear spaces, Trans. Amer. Math. Soc. vol. 60 (1946) pp. 519-537.

HARVARD UNIVERSITY 